Titolo: Il trattamento con interferone leucocitario + ribavirina in un paziente di 80 anni con $\mathrm{HCV}$ cronica genotipo 2, in fase di cirrosi compensata

Autori: Carbone R. ${ }^{1}$

Tipo: Rapporto breve

Keywords: Alfaferone;

\title{
Introduzione
}

Il trattamento dei pazienti anziani (over 65 anni), affetti da epatite cronica $C$, è da sempre stato oggetto di controversie scientifiche per la difficoltà a quantificare il reale rapporto rischio-beneficio del trattamento stesso.

Le domande che più frequentemente si pone il clinico, quando deve decidere in merito, sono relative agli eventuali vantaggi derivanti dall'eradicazione virale e in che percentuale questa possa favorevolmente influire in termini di aumento di sopravvivenza per riduzione di cirrosi scompensata e riduzione di comparsa di HCC (carcinoma epatocellulare), in pazienti anziani, con epatopatia in fase avanzata e fibrosi 3-4 secondo Metavir (misurata con Fibroscan) o Staging 4-6 alla biopsia epatica, secondo Ishak.

Questi dubbi hanno fortemente limitato l'approccio terapeutico al paziente con età $>65$ anni e molti colleghi non si sono di fatto dedicati a questa tipologia di pazienti, considerandoli, inoltre, troppo difficili da trattare e con scarsa risposta.

Occorre innanzitutto chiarire cosa si intenda per paziente "anziano". Senza entrare nel merito di concetti prettamente filosofici è però indubbio come il concetto di anzianità si sia profondamente modificato nel corso degli ultimi decenni. Durante i primi trattamenti con

\footnotetext{
Ambulatorio di Epatologia

Azienda Ospedaliera "SS. Antonio e Biagio e Cesare Arrigo" di Alessandria

mail: rcarbone@ospedale.al.it
} 
interferone (IFN), venivano considerati anziani e non eleggibili al trattamento, molti pazienti con età superiore a 60 anni e negli stessi studi registrativi delle terapie con IFN; la scheda tecnica riporta l'utilizzo del farmaco testato in un range di età 18-65 anni.

L'approccio al paziente $>65$ anni è stato pertanto fortemente limitato dalla giusta prudenza per le incognite sulla tolleranza al trattamento con IFN \pm ribavirina (RBV) e dalla scarsità di dati sui risultati del trattamento stesso in termini di raggiungimento della SVR nel paziente anziano con fibrosi avanzata e sugli effetti collaterali del trattamento.

Il risultato di questo prudente comportamento del clinico, nei confronti del paziente over 65 anni con $\mathrm{HCV}$ (Hepatitis $C$ virus) cronica, ha però condotto al risultato di trovare attualmente un numero rilevante di pazienti ultrasettantenni, senza particolari comorbilità $\mathrm{e}$ in buone condizioni generali fisiche e psichiche, ma con cirrosi compensata o comunque, con fibrosi avanzata oppure ricoverati per scompenso ascitico o per comparsa di HCC.

Nella mia personale esperienza in merito ho trattato, dal 1996 a oggi, oltre 80 pazienti over 65 anni con fibrosi avanzata o con cirrosi compensata HCV-correlata, con il risultato di avere ottenuto una SVR (sustained virological response) del 25\% nei pazienti con genotipo 1 e dell' $80 \%$ nel genotipo 2 .

Nel gruppo dei pazienti trattati, che hanno raggiunto la SVR, non si è registrato a tutt'oggi alcun caso di scompenso epatico e si è registrato un solo caso di HCC a distanza di 10 anni da una SVR ottenuta su fegato cirrotico. I drop-out sono stati inferiori al 3\% e si è potuta documentare una netta regressione della fibrosi epatica rilevata al fibroscan dopo il trattamento.

Il ricorso ai fattori di crescita granulocitari o alla eritropoietina è risultato sovrapponibile ai pazienti di età inferiore ai 65 anni. Altrettanto evidente è stato il risultato ottenuto sulla valutazione comparativa, prima e dopo il trattamento, dei valori di attività protrombinica, colinesterasi, sintesi del colesterolo, albuminemia, valore assoluto delle piastrine. Nel trattamento del paziente cirrotico over 65 , con genotipo virale 2 i miei risultati, in termini di SVR, sono stati, come già anticipato, dell' $80 \%$ rispettando però le condizioni di seguito indicate.

- Durata ottimale del trattamento: 24 o 48 settimane, a seconda della rapidità della risposta virologica.

- Dosaggio ottimale del trattamento.

- Gestione e correzione tempestiva degli effetti collaterali.

- Tolleranza soggettiva al trattamento, che permetta di portare a termine il trattamento stesso. 
Il gold standard, nel trattamento dell'epatopatia cronica HCV-correlata genotipo 2 e 3 è certamente rappresentato dall'associazione di INF peghilato (Peg-IFN)-alfa-2a o alfa-2b + $\mathrm{RBV}$, ma una delle cause possibili di interruzione di tale trattamento è proprio l'intolleranza soggettiva al Peg-IFN.

L'intolleranza spesso migliora spontaneamente con il proseguimento della terapia o con la riduzione posologica dell'IFN-alfa-2a, da $180 \mu \mathrm{g} / \mathrm{sett}$. a $135 \mu \mathrm{g} / \mathrm{sett}$. e dell'INF-alfa-2b a 1 $\mu \mathrm{g} / \mathrm{kg} / \mathrm{sett}$. A volte, però, la riduzione dell'INF non risolve il problema dell'intolleranza soggettiva. In questi precisi casi l'utilizzo dell'INF leucocitario rappresenta una preziosa risorsa, che permette di portare a termine la terapia, rispettandone i tempi previsti di trattamento.

\section{Il caso clinico}

Il paziente T.L. di 80 anni, italiano, accede per la prima volta presso il nostro Ambulatorio di Epatologia nel mese di novembre del 2011, in seguito al recente occasionale riscontro di positività all'HCV durante accertamenti preoperatori per un intervento di plastica aortica.

L'accertamento di altre patologie indicava un'ipertrofia prostatica.

Ottime risultavano le condizioni generali complessive, fisiche e psichiche del paziente, che è totalmente autonomo. Le terapie in atto sono: acido acetilsalicilico, tamsulosina, atenololo, enalapril da $5 \mathrm{mg}$. Viene completato il corretto inquadramento diagnostico.

\section{Esami di laboratorio e strumentali}

A febbraio del 2012, la situazione risulta come di seguito indicato.

- Epatopatia cronica HCV-correlata, genotipo 2, in cirrosi compensata.

- HCV-RNA: 1.848 .000 UI.

- AST e ALT aumentate di 1,5 i valori normali, con inversione del rapporto AST/ALT.

- Pregressa epatite A.

- Recettivo per HBV: praticata vaccinazione.

- Emocromo nella norma; PLT, nella norma.

- ETG addome con fegato aumentato di volume a struttura disomogenea e steatosica, e milza, nei limiti.

- Elastografia epatica, con riscontro di F 4 e Stiffness di 21,5 Kpa.

- Funzionalità renale, tiroidea, nella norma. 
- Autoanticorpi: assenti.

- Crioglobuline: assenti.

- Peso kg 74.00, H $170 \mathrm{~cm}$.

\section{La decisione terapeutica}

Il paziente, adeguatamente informato sullo stadio della patologia epatica e sulle problematiche connesse a un'eventuale terapia, risultava determinato ad affrontare il ciclo terapeutico con Peg-IFN-alfa-2b alla posologia di 1,5 $\mu \mathrm{g} / \mathrm{kg} / \mathrm{sett}$. + RBV $4 \mathrm{cp}$ die da $200 \mathrm{mg}$. La durata del piano terapeutico veniva fissata in 24 settimane, prorogabili eventualmente a 48 , sulla base della risposta virologica e della tolleranza.

\section{Il trattamento}

La terapia veniva iniziata a febbraio 2012 con Peg-IFN-alfa-2b 1,5 $\mu \mathrm{g} / \mathrm{kg} / \mathrm{settimana}+\mathrm{RBV}$ $800 \mathrm{mg} /$ die. Tolleranza soggettiva al trattamento da subito scarsa, non miglioramenti nel corso del trattamento stesso, pur senza particolari ripercussioni sulla crasi ematica, tranne una progressiva piastrinopenia, irrilevante sul rischio emorragico. Febbre, artromialgie, inappetenza e calo ponderale. Non tosse, non dermatite. Risposta virologica alla terapia, buona, ma non ottimale, senza raggiungere infatti la RVR. Gli effetti collaterali erano quindi imputabili esclusivamente al Peg-IFN.

Nella tabella I vengono riportati i principali parametri rilevati al baseline e dopo 4 settimane e 12 settimane, utilizzando Peg-IFN-alfa-2b +RBV.

Tabella I.

Principali parametri rilevati al basale e dopo 4 settimane dopo trattamento con Peg-IFN alfa- $2 b+R B V$.

\begin{tabular}{llll}
\hline $\begin{array}{l}\text { Parametri di } \\
\text { laboratorio }\end{array}$ & Al baseline & Dopo 4 settimane & Dopo 12 settimane \\
\hline $\begin{array}{l}\text { Globuli } \\
\text { bianchi }\end{array}$ & $5,000 \mathrm{mcl}$ & $4,300 \mathrm{mcl}$ & $4,200 \mathrm{mcl}$ \\
Globuli rossi & $5,300,000 \mathrm{mcl}$ & $4,150,000 \mathrm{mcl}$ & $4,096,000 \mathrm{mcl}$ \\
Emoglobina & $15,2 \mathrm{~g} / \mathrm{dl}$ & $12 \mathrm{~g} / \mathrm{dl}$ & $11,7 \mathrm{~g} / \mathrm{dl}$ \\
PLT & $229,000 \mathrm{mcl}$ & $108,000 \mathrm{mcl}$ & $88,000 \mathrm{mcl}$ \\
AST & $78 \mathrm{U} / \mathrm{I}(0-40)$ & $22 \mathrm{U} / \mathrm{I}$ & $24 \mathrm{U} / \mathrm{I}$ \\
ALT & $68 \mathrm{U} / \mathrm{I}(0-45)$ & $18 \mathrm{U} / \mathrm{I}$ & $19 / \mathrm{UI}$ \\
HCV-RNA & $128,000 \mathrm{UI} / \mathrm{ml}$ & $30 \mathrm{UI} / \mathrm{ml}$ & $<15 \mathrm{UI}$ \\
Peso kg & & 74 & 71 \\
\hline
\end{tabular}


Tolleranza soggettiva pessima, con astenia marcata e artromialgie diffuse, inappetenza e calo ponderale di $4 \mathrm{~kg}$ in 12 settimane. Febbre per 2 giorni dopo ogni somministrazione di PegIFN, pur ridotto dalla 8 a settimana di terapia a $1 \mu \mathrm{g} / \mathrm{kg} / \mathrm{settimana}$.

Non venendo raggiunta la risposta virologica precoce (RVR), ma avendo comunque raggiunto la completa negativizzazione della carica virale alla $12^{\mathrm{a}}$ settimana e trattandosi di un paziente con fibrosi avanzata, si proponeva di protrarre il trattamento per complessive 48 settimane.

In considerazione della cattiva tolleranza soggettiva alla terapia con Peg-IFN, veniva illustrata, al paziente, una diversa opzione curativa, con la sospensione del Peg-IFN stesso e il passaggio all'INF leucocitario, meglio tollerato, con la finalità di condurre a termine il programmato ciclo terapeutico di 48 settimane.

\section{Il cambio del Peg-IFN con IFN leucocitario}

Si proponeva quindi al paziente di modificare la terapia, sostituendo il Peg-IFN-alfa-2b, già utilizzato alla posologia ridotta di $1,0 \mu \mathrm{g} / \mathrm{kg} / \mathrm{settimana}$, con IFN leucocitario, $3 \mathrm{MU}$ a dì alterni (alfaferone), mantenendo la RBV al dosaggio precedente di $4 \mathrm{cp}$ die da $200 \mathrm{mg}$.

Veniva quindi redatto un nuovo piano terapeutico per la durata di 36 settimane, per completare le 48 settimane complessive di trattamento, in un paziente cirrotico, senza raggiungimento della risposta virologica precoce.

Tabella II

Principali parametri di laboratorio

(16a,24a e 48a settimana di terapia con alfaferone 3 MU a dì alterni+RBV 44 cp/die da 200 mg).

\begin{tabular}{llll}
\hline \multicolumn{1}{c}{$\begin{array}{c}\text { Parametri di } \\
\text { laboratorio }\end{array}$} & \multicolumn{1}{c}{$16^{\mathrm{a}}$ settimana } & \multicolumn{1}{c}{$24^{\mathrm{a}}$ settimana } & \multicolumn{1}{c}{$48^{\mathrm{a}}$ settimana } \\
\hline G.b. & $4,250 \mathrm{mcl}$ & $4,350 \mathrm{mcl}$ & $3,860 \mathrm{mcl}$ \\
G.R. & $4,176,000 \mathrm{mcl}$ & $4,250,000 \mathrm{mcl}$ & $3,650,000 \mathrm{mcl}$ \\
$\mathrm{Hb}$ & $11,2 \mathrm{~g} / \mathrm{dl}$ & $11,9 \mathrm{~g} / \mathrm{dl}$ & $11,4 \mathrm{~g} / \mathrm{dl}$ \\
$\mathrm{PLT}$ & $134,000 \mathrm{mcl}$ & $142,000 \mathrm{mcl}$ & $146,000 \mathrm{mcl}$ \\
AST/ALT & nei limiti, ma invertite & nei limiti, ma invertite & nei limiti, ma invertite \\
HCV-RNA & $<15 \mathrm{UI}$ & $<15 \mathrm{UI}$ & $<15 \mathrm{UI}$ \\
\hline
\end{tabular}

\section{Andamento della terapia e tolleranza al nuovo trattamento}

La tolleranza soggettiva alla nuova terapia con IFN leucocitario si mostrava da subito ottima e tale si manteneva nelle rimanenti 36 settimane di terapia, con scomparsa della febbre, delle artromialgie, miglioramento dell'astenia e dell'appetito, con progressiva ripresa del peso, pur senza raggiungere quello del baseline. Nella tabella II vengono riportati i valori dei principali 
parametri di laboratorio alla $16^{\mathrm{a}}, 24^{\mathrm{a}}$ e $48^{\mathrm{a}}$ settimana di terapia, con alfaferone $3 \mathrm{MU}$ a dì alterni + RBV 4 cp/die da 200 mg. terapia di 36 settimane con IFN leucocitario 3 MU a dì alterni + RBV 800 mg/die, terminava il 2 febbraio 2013 e iniziava il previsto follow-up semestrale.

Al controllo a distanza di 6 mesi dal termine del trattamento (agosto 2013) indicava i valori di seguito indicati.

- HCV-RNA: negativo.

- Risultato del trattamento: risposta virologica sostenuta (SVR).

- Elastografia epatica di controllo, passata da 21,5 Kpa (F 4) a 7,1 Kpa (F 1).

- ETG di controllo: fegato e milza, nei limiti, non lesioni focali.

\section{Conclusioni}

Il passaggio in corso di trattamento all'IFN leucocitario ha permesso di condurre a termine una terapia su un paziente cirrotico anziano, che diversamente avrebbe interrotto il trattamento precedentemente impostato, alla $12^{\mathrm{a}}$ settimana.

Le sole 12 settimane di trattamento con Peg-IFN+RBV, in un paziente con fibrosi avanzata, elevata replicazione virale al baseline (1.848.000 UI) e non raggiungimento della negativizzazione virologica alla $4^{\mathrm{a}}$ settimana di terapia, non potevano in alcun modo portare a una SVR. Tale favorevole risultato è stato ottenuto esclusivamente con il passaggio all'IFN leucocitario che, nel caso specifico essendo stato ottimamente tollerato, ha permesso di portare a termine un corretto trattamento di complessive 48 settimane.

Il follow-up del paziente proseguirà con un controllo semestrale della funzionalità epatica + alfa 1 fetoproteina + ETG (ecotomografia) addome superiore, come previsto per i pazienti con pregressa documentata fibrosi $\mathrm{F} 4$.

Tutta la documentazione relativa a questo caso clinico e ai restanti 79 pazienti trattati con IFN e RBV, secondariamente a un'epatopatia cronica HCV-correlata con fibrosi avanzata ed età $>65$ anni, è disponibile presso il mio archivio cartaceo e informatico, nell'Ambulatorio di Epatologia dell'Ospedale Civile di Alessandria e sarò lieto di fornirla a chiunque fosse interessato a unirla e confrontarla con altre analoghe esperienze per uno studio sul paziente "diversamente giovane" con epatite cronica C. 